\title{
Determination of the proton parton density functions at HERA
}

\author{
Juan TERRON* (on behalf of the $\mathrm{H} 1$ and ZEUS Collaborations) \\ Dept. de Física Teórica, Facultad de Ciencias, Universidad Autónoma de Madrid, E-28049 \\ Madrid, Spain \\ E-mail: terron@mail.desy.de
}

\begin{abstract}
Recent experimental progress on the determination of the parton distribution functions in the proton from the H1 and ZEUS Collaborations at HERA is reported. New QCD fits yielding improved determinations of the parton distribution functions in the proton are described. On one hand, precise measurements of neutral-current deep inelastic scattering at low $Q^{2}$ have allowed an improved determination of the gluon and sea distributions at low $x$. On the other hand, the high statistics measurements of high $Q^{2}$ neutral- and charged-current deep inelastic scattering data with electron and positron beams from HERA II help to improve the up-quark and down-quark valence distributions at high $x$ free from those corrections that affect fixed-target DIS data.
\end{abstract}

European Physical Society Europhysics Conference on High Energy Physics, EPS-HEP 2009,

July 16 - 222009

Krakow, Poland

${ }^{*}$ Speaker. 


\section{Introduction}

Deep inelastic scattering (DIS) of leptons off hadrons has been a unique source of information on the structure of the proton. Deep inelastic ep scattering is understood to proceed through the exchange of a highly virtual boson $\left(\gamma, Z^{\circ}\right.$ or $\left.W^{ \pm}\right)$between the electron and one of the constituents of the proton. The kinematics of the fully inclusive cross section for $e p \rightarrow e(v)+\mathrm{X}$ can be described by two independent kinematical variables such as $Q^{2}$, the virtuality of the exchanged boson, and Bjorken $x$, which, in the parton model, corresponds to the fractional momentum of the proton carried by the struck quark. Another useful variable is the inelasticity parameter $y$ given by $y=$ $Q^{2} / s x$, where $s$ is the squared $e p$ center-of-mass energy. At HERA, electrons or positrons of energy $27.5 \mathrm{GeV}$ collided with protons of $820(920) \mathrm{GeV}$ allowing an extension of the kinematic range by two orders of magnitude in both $Q^{2}$ and $x$ with respect to earlier fixed-target experiments.

The cross section for the neutral current (NC) DIS inclusive process can be described in terms of three structure functions as follows:

$$
\frac{d \sigma\left(e^{ \pm} p\right)}{d x d Q^{2}}=\frac{2 \pi \alpha^{2}}{x Q^{4}} \cdot\left(Y_{+} \cdot F_{2}\left(x, Q^{2}\right)-y^{2} \cdot F_{L}\left(x, Q^{2}\right) \mp Y_{-} \cdot x F_{3}\left(x, Q^{2}\right)\right),
$$

where $Y_{ \pm}=1 \pm(1-y)^{2}$. The structure function $F_{2}$ is dominant and at leading order (LO) in QCD and for $Q^{2} \ll M_{Z}^{2}$ is given by the sum of the quark and antiquark densities weighted by the squared electric charges of the quarks, $F_{2}=x \sum_{i} e_{i}^{2}\left(q_{i}+\bar{q}_{i}\right)$. The longitudinal structure function, $F_{L}$, is only relevant at high $y$ and is predicted to be zero at LO in QCD. The structure function $x F_{3}$, which violates parity, becomes significant only at high $Q^{2}$. As a result, NC DIS represents a clean probe of the parton distribution functions (PDFs) in the proton.

The LO QCD cross sections for the charged current (CC) DIS inclusive processes are

$$
\frac{d \sigma\left(e^{+} p\right)}{d x d Q^{2}}=\frac{G_{F}^{2}}{2 \pi} \eta_{W}^{2} \cdot \sum_{i}\left(\bar{u}_{i}+(1-y)^{2} d_{i}\right) \text { and } \frac{d \sigma\left(e^{-} p\right)}{d x d Q^{2}}=\frac{G_{F}^{2}}{2 \pi} \eta_{W}^{2} \cdot \sum_{i}\left(u_{i}+(1-y)^{2} \bar{d}_{i}\right),
$$

where the sums run over all (anti-)up-type and (anti-)down-type quarks and $\eta_{W}=M_{W}^{2} /\left(Q^{2}+M_{W}^{2}\right)$. These cross sections depend upon different combinations of PDFs than NC DIS and provide a handle on the flavour content of the proton.

The importance of a precise determination of the proton PDFs lies in the fact that the prediction of the Standard Model (SM) for any hard process at a hadron collider (such as the LHC) has the form of a convolution of the matrix elements with the proton PDFs. An accurate prediction requires a precise determination of the proton PDFs. The proton PDFs in most of the region of the kinematic plane that will be probed at the LHC can be determined from HERA data by using the DGLAP evolution equations in $Q^{2}$. For instance, the cross section for Higgs production through the gluongluon fusion process is sensitive to the gluon density at $x \sim 8 \cdot 10^{-3}$ and $Q^{2} \sim 13000 \mathrm{GeV}^{2}$ for $m_{H}=115 \mathrm{GeV}$ while the cross section for single $W$ production is sensitive to the sea distribution at $x \sim 6 \cdot 10^{-3}$ and $Q^{2} \sim 6400 \mathrm{GeV}^{2}$ [1].

\section{H1 QCD fit}

One of the fundamental inputs in an extraction of the proton PDFs is the measurement of the doubly differential cross section in $x$ and $Q^{2}$ for NC DIS. The H1 collaboration has recently 


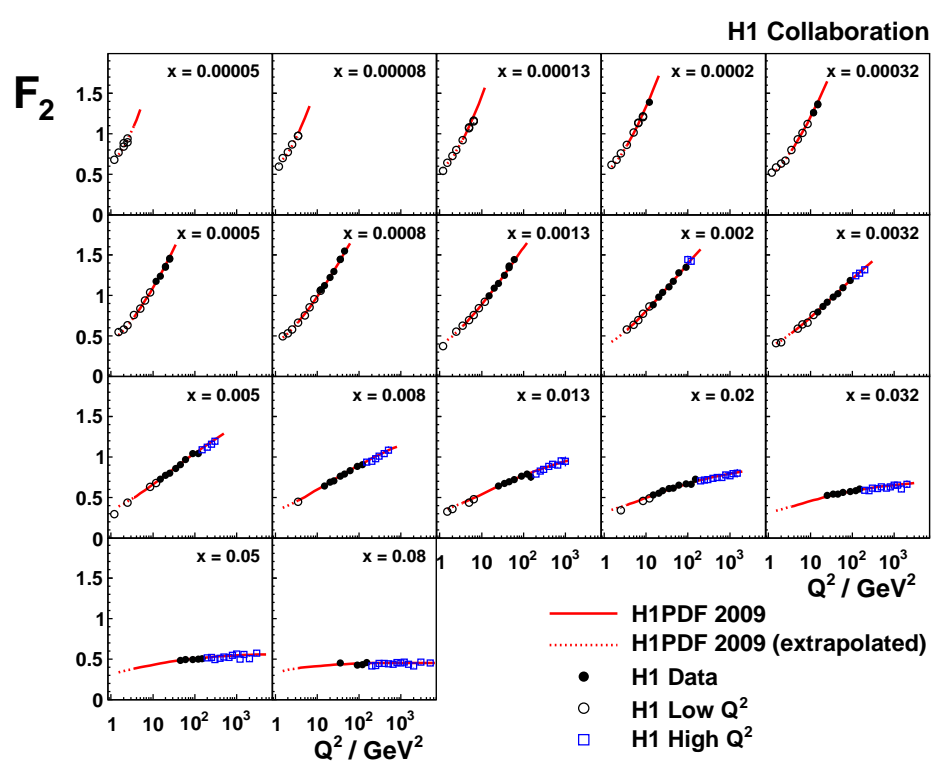

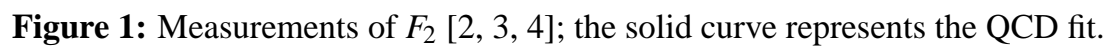

published [2] the most precise determination of this quantity in the region of medium $Q^{2}, 12<$ $Q^{2}<150 \mathrm{GeV}^{2}$. Values of $F_{2}$ have been extracted from the reduced cross section by correcting for the $F_{L}$ contribution as given by the QCD fit (see below) and the total uncertainty amounts to 1.3-2\%. These new measurements of $F_{2}$ [2] together with those at lower and higher $Q^{2}$ [3, 4] are shown in Fig. 1 as a function of $Q^{2}$ for fixed values of $x . \quad F_{2}$ provides direct information on the quark densities through the relation $F_{2}=x \sum_{i} e_{i}^{2}\left(q_{i}+\bar{q}_{i}\right)$. Furthermore, it also provides indirect information on the gluon density: the large and positive scaling violations observed at low $x$ are understood to arise from the dominance of the boson-gluon fusion process and, thus, are sensitive to the gluon content of the proton.

The determination of the proton PDFs is based on the DGLAP evolution equations at next-toleading order (NLO) in $\alpha_{s}$; these equations dictate the $Q^{2}$-dependence of the PDFs in terms of the quark and gluon densities, $\alpha_{s}$ and the splitting functions. These equations yield the proton PDFs at any value of $Q^{2}$ provided they are input as functions of $x$ at some input scale $Q_{0}^{2}$. In order to determine the proton PDFs additional experimental information besides that provided by $F_{2}$ is needed on the quark densities at high $x$ and the flavour composition of the sea. In the new QCD fit by H1, H1PDF2009 [2], which makes use only of $\mathrm{H} 1$ data, the additional data sets are the measurements of NC and CC DIS at high $Q^{2}$ [4, 5]. The two major improvements with respect to earlier H1 QCD fits are 1) the inclusion of the precise measurement of NC DIS for $Q^{2}<150 \mathrm{GeV}^{2}$ [2, 3], and 2) the improved theoretical treatment of heavy quarks provided by the VFNS scheme of Roberts and Thorne [6].

In the H1 QCD fit five combinations of proton PDFs are selected: the up-quark valence $\left(x u_{V}\right)$, the down-quark valence $\left(x d_{V}\right)$, the gluon $(x g)$, the total anti-up $(x \bar{U}=x \bar{u}+x \bar{c})$ and the total antidown $(x \bar{D}=x \bar{d}+x \bar{s}+x \bar{b})$ distributions. They are parametrised at $Q_{0}^{2}=1.9 \mathrm{GeV}^{2}$ by the functional form $x f(x)=A_{p} x^{B_{p}}(1-x)^{C_{p}}\left(1+D_{p} x+E_{p} x^{2}\right)$ and constraints are applied to the parameters $A_{p}$ and $B_{p}$ (see [2] for details). There are in total 10 free parameters and $\alpha_{s}\left(M_{Z}\right)$ is fixed to 0.1176 . 

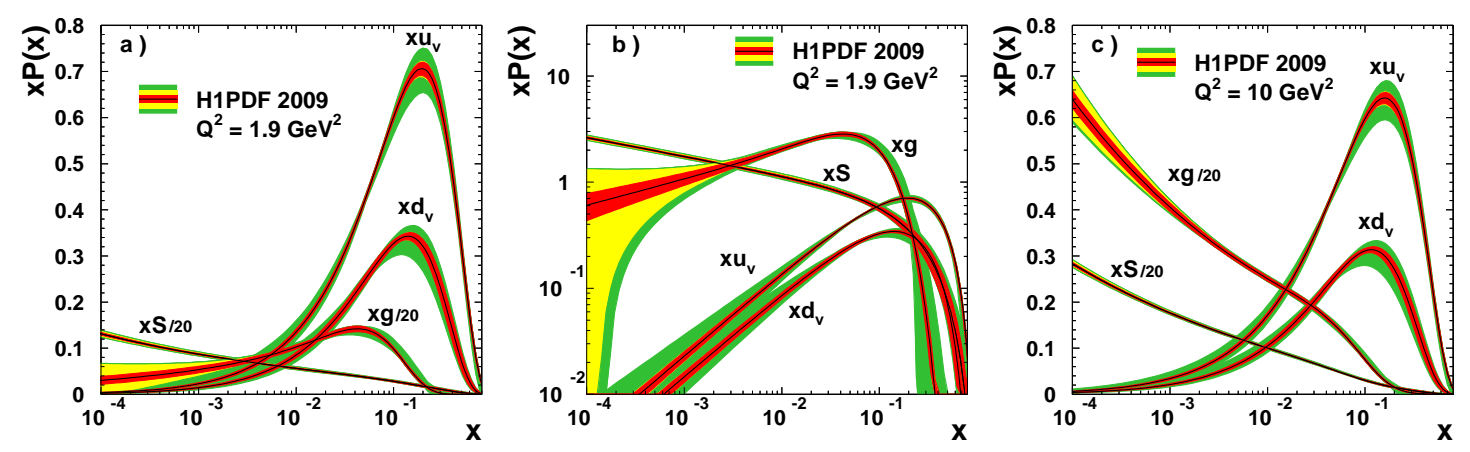

Figure 2: PDFs as determined by the H1PDF2009 QCD fit at $Q^{2}=1.9 \mathrm{GeV}^{2}$ (a,b) and $10 \mathrm{GeV}^{2}$ (c).

Additional conditions are further applied: $F_{2} \geq 0$ and $F_{L} \geq 0$; all PDFs $\geq 0$ and the valence should not be too low compared to the sea at high $x$; the last two conditions are not applied in the estimation of the parametrisation uncertainty.

In the H1 QCD fit all data points with $Q^{2}$ above $Q_{\text {min }}^{2}$ of $3.5 \mathrm{GeV}^{2}$ are included. The QCD fit gives a good description of all data with $\chi^{2} / n d o f=587 / 644$. The resulting PDFs as functions of $x$ for two scales $\left(Q^{2}=1.9\right.$ and $\left.10 \mathrm{GeV}^{2}\right)$ are shown in Fig. 2 together with the experimental uncertainty (the red shaded band), the model uncertainty (the yellow shaded band) and the parametrisation uncertainty (the green shaded band). The experimental uncertainty has been obtained from the criterion $\Delta \chi^{2}=1$ using the Hessian method. The model uncertainty has been obtained by varying the charm mass, the bottom mass, the suppression factor of the strange sea, $Q_{\min }^{2}$ and $Q_{0}^{2}$. The latter represents the major source of uncertainty at low $x$ (see Fig. $2 \mathrm{~b}$ ). The parametrisation uncertainty has been estimated by considering alternative parametrisations leading to unphysical behaviour at large $x$ but which still give good fits to the data. These new determinations have reduced uncertainties at low $x$ with respect to the previous fit (H1PDF 2000 [凹) and larger (and more realistic) uncertainties at high $x$, which are dominated by the newly-estimated parametrisation uncertainty. It is observed that at the input scale the sea quarks dominate at low $x$ and the gluon density has a valence-like shape (see Fig. Za), whereas at $Q^{2}=10 \mathrm{GeV}^{2}$ the gluon dominates at low $x$ and the rise towards low $x$ is similar for gluons and quarks (see Fig. 国).

\section{ZEUS QCD fit}

In the ZEUS QCD fit [7] five combinations of proton PDFs are selected: $x u_{V}, x d_{V}, x g$, the total sea $(x S)$ and $x \Delta=x(\bar{d}-\bar{u})$. They are parametrised at $Q_{0}^{2}=7 \mathrm{GeV}^{2}$ by the functional form $x f(x)=$ $p_{1} x^{p_{2}}(1-x)^{p_{3}}\left(1+p_{4} x\right)$ and constraints are applied on the parameters $p_{i}$. The VFNS scheme of Roberts and Thorne is used for the treatment of heavy quarks. There are in total 11 free parameters and $\alpha_{s}\left(M_{Z}\right)$ is fixed to 0.118 . The evolution of the PDFs with the energy scale is performed using the DGLAP equations at NLO. The new ZEUS QCD fit represents an improvement with respect to the previous fit (ZEUS-JETS PDF [8]) due to the inclusion of new data sets: 1) the high $Q^{2}$ ZEUS $e^{-} p$ NC and CC data [9] from HERA II, with the aim of improving $x u_{V}$ at high $x$; 2) the high $Q^{2}$ ZEUS $e^{+} p$ CC [10] data from HERA II, with the aim of improving $x d_{V}$ at high $x$; and 3) the low $Q^{2}$ ZEUS NC data taken with different proton-beam energies (920, 575 and $460 \mathrm{GeV}$ ) from HERA II, with the aim of improving the gluon and sea distributions at low $\mathrm{x}$. 
ZEUS

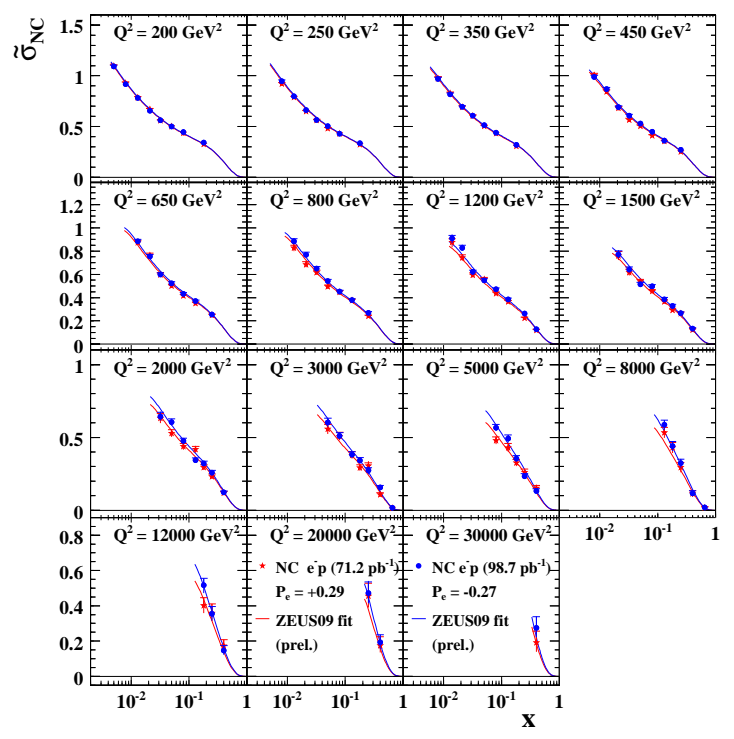

ZEUS

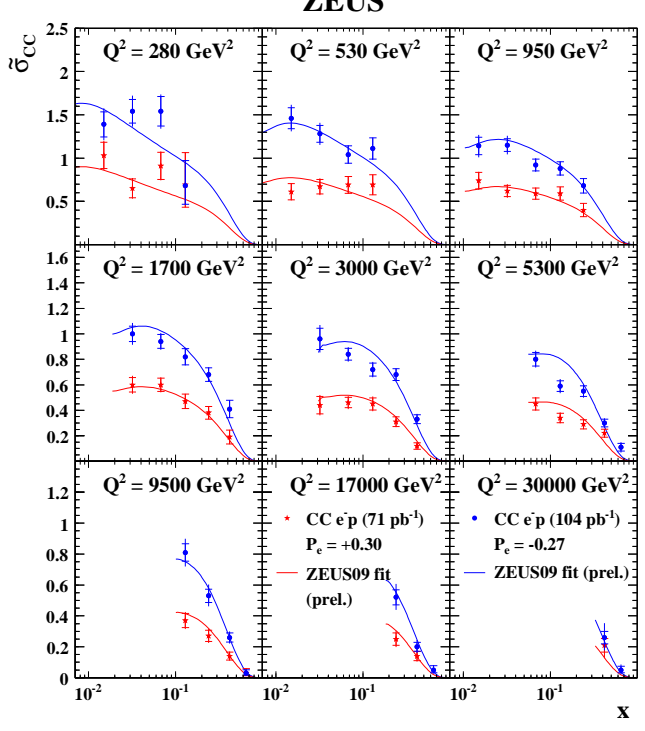

Figure 3: ZEUS $e^{-} p$ NC (left) and CC (right) data [9] from HERA II; the predictions of the ZEUS09 QCD fit are superimposed.

The new ZEUS QCD fit, ZEUS09, gives a good description of all the data sets ${ }^{1}$ with a total of 1060 data points and $\chi^{2} / n d o f=0.97$. In the fit full account of the correlated experimental uncertainties is taken by using the offset method. The results of the fit are compared in Fig. 3 (left) to the reduced cross sections for $e^{-} p$ NC DIS with longitudinally polarised $e^{-}$beams as a function of $x$ for fixed values of $Q^{2}$. These data provide a constrain on $x u_{V}$ at high x. A polarisation dependence is observed at high $Q^{2}$, which is understood to arise from the $\gamma-Z^{\circ}$ interference and the $Z^{\circ}$-exchange contribution, and is well described by the fit. This comparison constitutes a confirmation of the predictions of the electroweak sector of the SM in a space-like process.

The measurements of the double differential reduced cross section for $e^{-} p$ CC DIS as a function of $x$ for fixed values of $Q^{2}$ and for negative (blue dots) and positive (red dots) longitudinal polarisations of the electron beam are shown in Fig. 远 (right). As emphasized earlier, these data are sensitive to a different combination of PDFs than NC DIS and thus provide information on the flavour content of the proton. Most importantly, these data are sensitive to $x u_{V}$ at high $\mathrm{x}$. The results of the fits (red and blue lines) give a good description of the data and represent a confirmation of the electroweak predictions of the SM. Likewise the measurements of the double differential reduced cross section for $e^{+} p$ CC DIS (not shown) provides information on $x d_{V}$ at high $\mathrm{x}$.

The proton PDFs as functions of $x$ at $Q^{2}=10 \mathrm{GeV}^{2}$ are shown in Fig. $\bigoplus$ (left) together with the estimated uncertainties. They are consistent with the previous QCD fit, but have smaller uncertainties. The impact of the new data sets is illustrated in terms of the relative uncertainties of the PDFs as functions of $x$ at $Q^{2}=10 \mathrm{GeV}^{2}$ in Fig. $\bigoplus$ (right): the previous fit, ZEUS-JETS PDF, as the blue band, and the new fit, ZEUS09, as the yellow band. Significant improvements are obtained in the up-quark and down-quark valence at high $x$ free from nuclear corrections, higher twists and

\footnotetext{
${ }^{1}$ The ZEUS09 and ZEUS-JETS QCD fits include jet cross section measurements in NC DIS and photoproduction.
} 

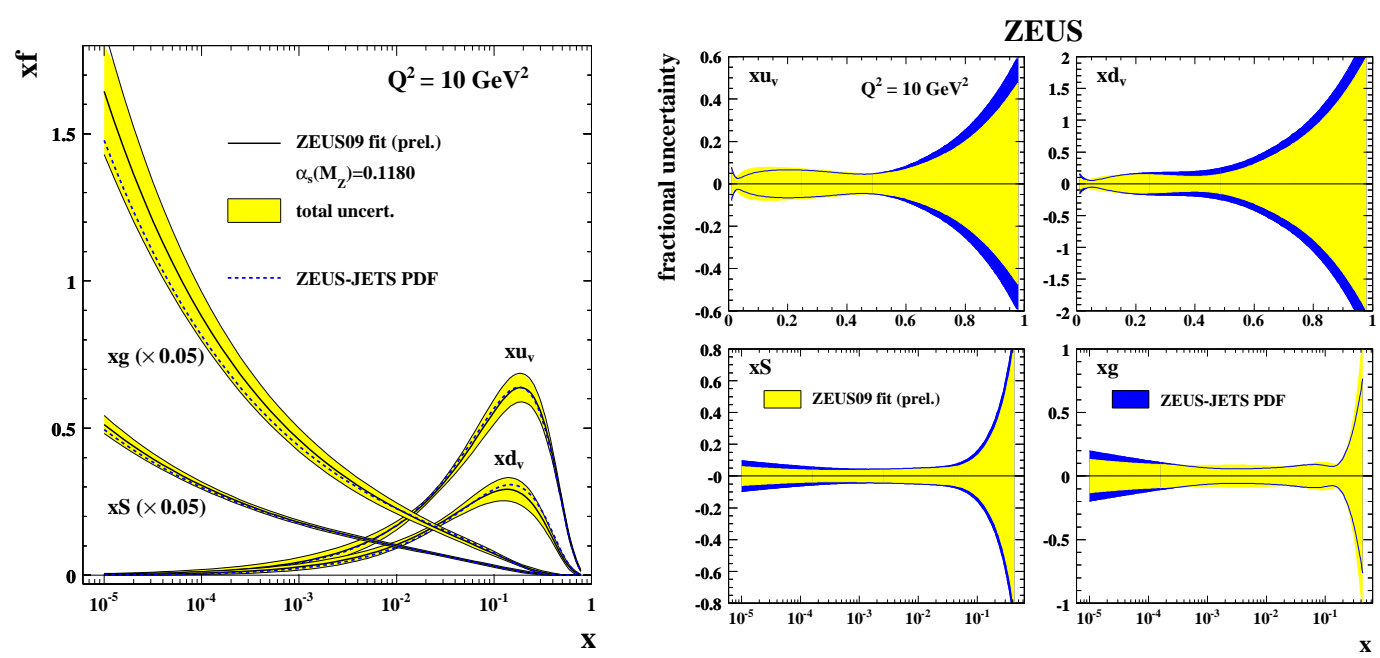

Figure 4: PDFs as determined by the ZEUS09 QCD fit at $Q^{2}=10 \mathrm{GeV}^{2}$ (left); relative uncertainties of the ZEUS09 PDFs compared to those of the ZEUS-JETS PDFs (right).

isospin symmetry assumptions.

\section{Summary}

In summary, the H1 and ZEUS collaborations have made new QCD fits yielding improved determinations of the proton PDFs. On one hand, precise measurements of NC DIS at low $Q^{2}$ have allowed an improved determination of the gluon and sea distributions at low $x$. On the other hand, the high statistics measurements of high $Q^{2} \mathrm{NC}$ and CC DIS data with electron and positron beams from HERA II help to improve the up-quark and down-quark valence distributions at high $x$ free from those corrections that affect fixed-target DIS data.

\section{References}

[1] J.M. Campbell, J.W. Huston and W.J. Stirling, Rept. Prog. Phys. 70 (2007) 89.

[2] H1 Coll., F.D. Aaron et al., DESY preprint DESY-09-005 [hep-ex/ 0904 . 3513].

[3] H1 Coll., F.D. Aaron et al., DESY preprint DESY-08-171 [hep-ex/ 0904 .0929].

[4] H1 Coll., C. Adloff et al., Eur. Phys. J. C30 (2003) 1.

[5] H1 Coll., C. Adloff et al., Eur. Phys. J. C13 (2000) 609 and Eur. Phys. J. C19 (2001) 269.

[6] R.S. Thorne and R.G. Roberts, Phys. Rev. D57 (1998) 6871; R.S. Thorne, Phys. Rev. D73 (2006) 054019 .

[7] ZEUS Coll., S. Chekanov et al., ZEUS-prel-09-010.

[8] ZEUS Coll., S. Chekanov et al., Eur. Phys. J. C42 (2005) 1.

[9] ZEUS Coll., S. Chekanov et al., Eur. Phys. J. C62 (2009) 625 and Eur. Phys. J. C61 (2009) 223.

[10] ZEUS Coll., S. Chekanov et al., ZEUS-prel-09-002. 